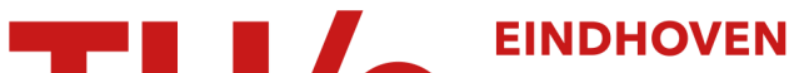 \\ UNIVERSITY OF \\ TECHNOLOGY
}

\section{Self-consistent field lattice model for polymer networks}

Citation for published version (APA):

Tito, N. B., Storm, C., \& Ellenbroek, W. G. (2017). Self-consistent field lattice model for polymer networks. Macromolecules, 50(24), 9788-9795. https://doi.org/10.1021/acs.macromol.7b01284

DOI:

10.1021/acs.macromol.7b01284

Document status and date:

Published: 05/12/2017

\section{Document Version:}

Publisher's PDF, also known as Version of Record (includes final page, issue and volume numbers)

\section{Please check the document version of this publication:}

- A submitted manuscript is the version of the article upon submission and before peer-review. There can be important differences between the submitted version and the official published version of record. People interested in the research are advised to contact the author for the final version of the publication, or visit the $\mathrm{DOI}$ to the publisher's website.

- The final author version and the galley proof are versions of the publication after peer review.

- The final published version features the final layout of the paper including the volume, issue and page numbers.

Link to publication

\section{General rights}

Copyright and moral rights for the publications made accessible in the public portal are retained by the authors and/or other copyright owners and it is a condition of accessing publications that users recognise and abide by the legal requirements associated with these rights.

- Users may download and print one copy of any publication from the public portal for the purpose of private study or research.

- You may not further distribute the material or use it for any profit-making activity or commercial gain

- You may freely distribute the URL identifying the publication in the public portal.

If the publication is distributed under the terms of Article $25 \mathrm{fa}$ of the Dutch Copyright Act, indicated by the "Taverne" license above, please follow below link for the End User Agreement:

www.tue.nl/taverne

Take down policy

If you believe that this document breaches copyright please contact us at:

openaccess@tue.nl

providing details and we will investigate your claim. 


\title{
Self-Consistent Field Lattice Model for Polymer Networks
}

\author{
Nicholas B. Tito, ${ }^{* \dagger, \ddagger(i) ~ C o r n e l i s ~ S t o r m, ~}{ }^{\dagger, \ddagger}$ and Wouter G. Ellenbroek ${ }^{\dagger, \ddagger(0)}$
}

${ }^{\dagger}$ Department of Applied Physics, Eindhoven University of Technology, PO Box 513, 5600 MB, Eindhoven, The Netherlands

${ }^{\ddagger}$ Institute for Complex Molecular Systems, Eindhoven University of Technology, PO Box 513, 5600 MB, Eindhoven, The Netherlands
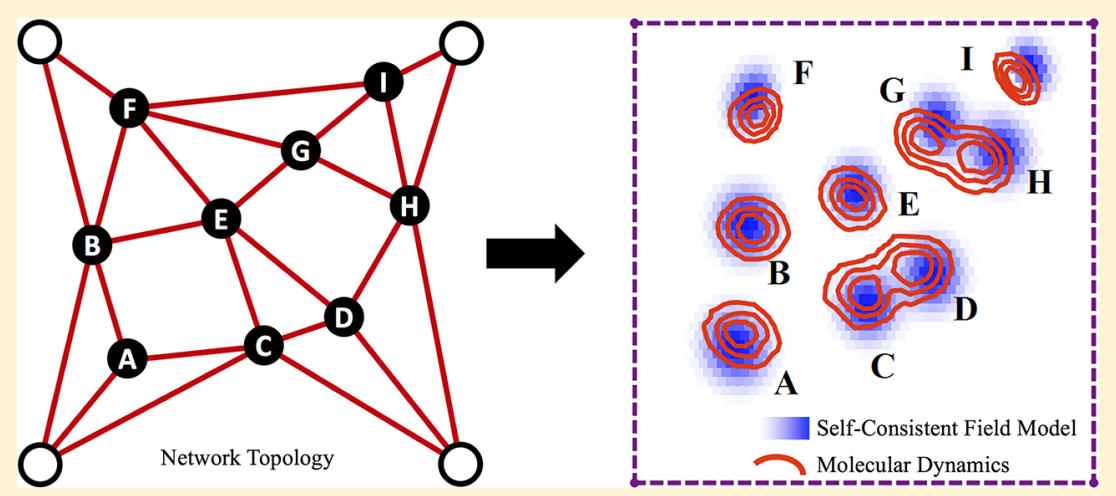

ABSTRACT: A lattice model based on polymer self-consistent field theory is developed to predict the equilibrium statistics of arbitrary polymer networks. For a given network topology, our approach uses moment propagators on a lattice to selfconsistently construct the ensemble of polymer conformations and cross-link spatial probability distributions. Remarkably, the calculation can be performed "in the dark", without any prior knowledge on preferred chain conformations or cross-link positions. Numerical results from the model for a test network exhibit close agreement with molecular dynamics simulations, including when the network is strongly sheared. Our model captures nonaffine deformation, mean-field monomer interactions, cross-link fluctuations, and finite extensibility of chains, yielding predictions that differ markedly from classical rubber elasticity theory for polymer networks. By examining polymer networks with different degrees of interconnectivity, we gain insight into cross-link entropy, an important quantity in the macroscopic behavior of gels and self-healing materials as they are deformed.

\section{INTRODUCTION}

Polymer networks are materials comprising macromolecules connected together by entanglements or cross-links. Depending on the chemistry involved, the polymers can be flexible or semiflexible, and the number of chains attached to each crosslink can be fixed or variable.

Polymeric networks have a wide range of applications in synthetic and natural settings. For example, when swollen by solvent into a gel, they are used as medical implants, artificial tissues, or flexible materials. ${ }^{1}$ Many structural biological materials, such as the intracellular cytoskeleton and the extracellular matrix, are meshworks of linked protein polymers; the unique mechanical functionalities of the fibers as well as the links between them often serve as inspiration for new material paradigms. Biological motifs have propelled the development of synthetic polymer networks as the basis for a variety of exciting new materials that self-heal when damaged, ${ }^{2,3}$ respond to stimuli, ${ }^{4}$ undergo microscopic adaptation when macroscopically deformed, ${ }^{5,6}$ or flow and reconfigure in situ upon temperature change.

Since the macroscopic behavior of polymer networks is almost always dominated by the entropy associated with the huge number of conformations each polymer chain can have, predicting their thermodynamic and mechanical properties requires a reliable sampling of the ensemble of microscopic states in the system. Thus, modeling approaches are deeply rooted in statistical mechanical models of the behavior of the individual polymers (see ref 8 for a comprehensive review). The two most common simulational approaches at the molecular scale are molecular dynamics (MD) and Monte Carlo (MC). They can be used to explore the evolution of microscopic structure in the network at equilibrium and upon deformation. ${ }^{9,10}$

Molecular dynamics, in particular, is naturally suited to the nature of the questions one would like to ask to elucidate the relations between microscopic organization, polymer (in)flexibility, geometry, connectivity, and dynamical mechanical response for typical polymer networks, but unfortunately the typical combination of large molecular weight, high densities, and large stiffness is prohibitive: Well-resolved MD simulations struggle to capture length scales beyond a couple of tens of mesh sizes.

Received: June 16, 2017

Revised: November 18, 2017

Published: December 5, 2017 
Monte Carlo approaches have been able to capture larger scale response but lack realistic dynamics-a deficiency that hybrid MC-Langevin approaches ${ }^{11}$ have been able to address only in part. These and other shortcomings have led to the development of simplified models, ${ }^{12,13}$ often on lattices. ${ }^{14-17}$

What most of these approaches (other than full MD) share in common is an explicit separation of cross-links and polymers, treating the sections of polymer between cross-links as springlike components and minimizing their summed (free) energy over the positions of the cross-links connecting them. This works well when the cross-links themselves are fixed in space, but in reality these flucutuate as much as if not more than other components of the network.

To circumvent the codependency of polymer distributions and those of the cross-linkers, one common approach has been to assume that the cross-links in the polymer network deform affinely with the material; this leads to simple predictions for the free energy of the material as it is deformed-the "classical rubber elasticity theory". ${ }^{18}$ However, the positional entropy of the cross-links themselves plays a crucial role in the behavior of the material, ${ }^{19}$ particularly for marginal and submarginal networks where cross-link fluctuations can grow much larger than the network mesh size. ${ }^{20}$ These fluctuations lead to, at times, nonintuitive trends. For example, a surface-grafted polymer network can exhibit a smaller shear modulus than an equivalent polymer brush. ${ }^{21}$ Cross-link fluctuations can be approximated using the "phantom network model", an improvement to the classical elasticity model. ${ }^{18}$ However, the model does not account for finite extensibility of the polymers, nor interactions with a spatially varying monomer density field.

In this work, we develop a lattice-based approach to crosslinked polymer networks that permits quick and reliable sampling of the full configurational space, including the positional fluctuations and nonaffine displacements of crosslinks. Lattice self-consistent field theory (SCFT) for polymers ${ }^{22}$ is invoked to approximate the equilibrium spatial distributions of the polymers and cross-links given the input network topology. The polymers themselves are phantom chains interacting with a spatially varying mean monomer density field, and the network topology is enforced via additional spatially resolved self-consistent mean fields representing the cross-link distributions. The model takes as input the number of segments in the polymer strands connecting the cross-links and the network topology itself. Importantly, knowledge of the spatial arrangement of the cross-links is not needed a priori-in fact, these distributions are a central result of the model.

Schmid $^{23}$ has previously written self-consistent mean-field equations for approximating the equilibrium distribution of a permanently cross-linked polymer network. We invoke a similar approach here, developing the equations into explicit forms within the context of Scheutjens-Fleer lattice SCFT. ${ }^{22}$ This allows for a number of advances in practical application of the theory. Our approach converges on the equilibrium spatial distributions of cross-links (nodes) and polymer conformations without any prior knowledge of what these distributions are. The model also naturally incorporates finite extensibility of the polymer strands as the network is strongly deformed and can be easily extended to examine networks of semiflexible polymers. $^{24}$

A recent study developed an SCFT approach for examining the thermodynamics of micelle-like "nodes" in self-assembled networks of telechelic polymers. ${ }^{25}$ However, an approximation in their approach is that the spatial arrangement of the nodes is preimposed. This restricts the allowed configurations of the system, leading to an underestimation of the entropy, which can only be corrected by allowing the nodes to fluctuate as we do in the present work.

Our approach is applicable to "user-defined" network structures and can also incorporate boundary conditions (i.e., network nodes that are fixed in space as tether points). A spatially varying mean monomer density field is explicitly included in our mathematical derivation of the model, though in the examples we study here we restrict our attention to networks of noninteracting polymers. We illustrate derivation of the model in two dimensions; extension to three dimensions is trivial. The model presently lacks the ability to account for chain noncrossing and entanglements; suggestions for how to include this feature are given in the Conclusions section.

\section{MODEL}

Consider a two-dimensional polymer network having $J$ nodes connected by polymer strands or "bridges". Each bridge $b$ is composed of $N_{b}$ segments. The topology of the network is defined by specifying which nodes $j$ are connected to other nodes $k$ and the length $N_{b}$ of the bridge making each of these connections. Some of the nodes are fixed in space, serving as "anchor points", while the remaining nodes are allowed to fluctuate freely. The polymer network is represented on a twodimensional lattice; in this study, we choose a hexagonal lattice with six nearest neighbors per site, but any lattice can be used as long as the number of neighbors at each site is known. The lattice constant is taken to be unity, equal to the diameter of a polymer segment (either a Kuhn segment or a single monomer).

Polymer conformations for the bridges are generated on the lattice by the method of propagators in self-consistent mean fields, ${ }^{26,27}$ similar to the method of Scheutjens and Fleer. ${ }^{22} \mathrm{~A}$ propagator generates the conformational probability distribution of a lattice polymer, given a probability distribution $p_{1}(i)$ that the first segment is located at lattice site $i$. The Boltzmann weight for initiating the propagator at $i$ is given by

$$
W_{1}(i)=p_{1}(i) w(i)
$$

where $w(i)$ is the weight for placing a monomer at $i$. This weight may depend on any external field and is typically used to represent the effect of mean-field monomer-monomer interactions by taking $w(i)=\exp [-\beta \epsilon \rho(i)]$. Here, $\epsilon$ is the monomer-monomer interaction energy, $\beta=1 / k_{\mathrm{B}} T$, and $\rho(i)$ is the ensemble-average monomer density in site $i$ (to be obtained self-consistently). In this work, we will only examine cases where $\epsilon=0$ such that the lattice polymers are noninteracting; however, we include this term to show how it is incorporated into the model mathematically.

For subsequent segments $m=2, \ldots, N$, where $N$ is the number of segments comprising the polymer, the weight for pathways of $m$ steps that terminate at site $i$ is calculated by

$$
W_{m}(i)=w(i) \sum_{\left\{i_{\text {adj }}\right\}} W_{m-1}\left(i_{\text {adj }}\right)
$$

Here, $\left\{i_{\text {adj }}\right\}$ is the set of nearest neighbors to $i$ on the lattice, and $i_{\text {adj }}$ is one such neighbor. (Note that eq 2 represents a first-order Markov chain. Semiflexibility of the polymers can be incorporated by evolving the polymer configurations via a second-order Markov chain along with appropriate statistical weights for chain bending. ${ }^{24}$ ) 
Equation 2 is computed recursively for steps $m=2$ to $N$ of the propagator. At the edges of the lattice, reflecting boundary conditions are utilized ${ }^{22}$ (though other boundary conditions, e.g., periodic or absorbing, may also be used). The quantity $W_{N}(i)$ thus represents the weight for propagator pathways that terminate at $i$ in $N$ steps, given the distribution of propagator starting positions $p_{1}(i)$.

Equations 1 and 2 can also be calculated in reverse, starting from the $m=N$ side of the polymer given a distribution $p_{N}(i)$ of locations for monomer $N$ of the polymer. Using the composition law of propagators, ${ }^{27}$ the weight for finding segment $m$ of the polymer at $(i)$, given both the starting and ending point distributions $p_{1}(i)$ and $p_{N}(i)$, is

$$
Q_{m}(i)=\frac{W_{m}(i) W_{N-m+1}^{\prime}(i)}{w(i)}
$$

Here, $W$ and $W^{\prime}$ are the propagators starting from the $m=1$ and $m=N$ sides of the chain, respectively. (The factor of $w(i)$ in the denominator of eq 3 is to prevent double-counting the weight for placing a monomer at $i$, contained in both $W$ and $W^{\prime}$.)

Each polymer in the system is a bridge $b$ having $N_{b}$ segments, connecting two nodes $j$ and $k$. Suppose the first node $j$ has a probability "cloud" (spatial distribution) given by $P_{j}(i)$. The first segment of bridge $b$ is therefore located at $i$ with probability $p_{1}(i)=P_{j}(i)$. The statistical weight for terminating bridge $b$ at site $i^{\prime}$ is thus $W_{N_{b}}\left(i^{\prime}\right)$.

The bridge $b$ ultimately connects to node $k$. However, $W_{N_{b}}(i)$ is not the cloud for node $k$; it must be calculated as the intersection of all bridges $b_{1}, b_{2}, \ldots, b_{l}, \ldots, b_{n}$ that connect to $k$, where $n$ is the number of neighbors to node $k$. The end segment distribution $W_{N_{b}, l}(i)$ is calculated for each of these bridges $b_{l}$ via eq 2 , given the unique clouds $P_{j}(i)$ for each of the origin nodes. The cloud for node $k$ is then obtained by

$$
P_{k}(i)=\frac{1}{w(i)^{n-1}} \frac{\prod_{l=1}^{n} W_{N_{b, l}}(i)}{\sum_{i^{\prime}} \prod_{l=1}^{n}\left(W_{N_{b, l}}\left(i^{\prime}\right) / w\left(i^{\prime}\right)^{n-1}\right)}
$$

where the products are over all bridges $b_{l}$ that connect to node $k$, and the sum is over all lattice sites $i^{\prime}$ in the system. Dividing by $w(i)^{n-1}$ ensures that the weight for site $i$ is only counted once, as all bridges $n$ converge to only one segment (node) located at that position. The quantity $P_{k}(i)$ is therefore the (normalized) spatial probability distribution for node $k$ in the system.

Equation 4 represents a Bragg-Williams approximation to the real spatial probability distribution of node $k$. This is because in each microstate of the network the position of node $k$ depends on the position of its neighbors, which in turn depend on the positions of their neighbors, and so on. For node $k$ (with $n$ neighbors indexed by $l$ ) the ensemble probability of observing a particular set of spatial coordinates $i_{l}$ of these neighbors is a composite quantity $P\left(i_{1}, i_{2}, \ldots, i_{l}, \ldots, I_{n}\right)$. We would then compute the spatial distribution of node $k$ via

$$
P_{k}(i)=\frac{\text { const }}{w(i)^{n-1}} \sum_{i_{1}} \ldots \sum_{i_{n}}\left[P\left(i_{1}, i_{2}, \ldots, i_{l}, \ldots, i_{n}\right)\left(\prod_{l=1}^{n} K_{N_{b, l}}\left(i_{l}, i\right)\right)\right]
$$

where the nested sums are over all positions of all neighboring nodes to $k$, and $K_{N_{b}, l}(i, i)$ is the sum of propagator weights for going from site $i_{l}$ to site $i$ in $N_{b, l}$ segments. In principle, this can be computed; however, to make the method numerically tractable, we approximate

$$
P\left(i_{1}, i_{2}, \ldots, i_{l}, \ldots, i_{n}\right) \approx P_{1}\left(i_{1}\right) P_{2}\left(i_{2}\right) \ldots P_{l}\left(i_{l}\right) \ldots P_{n}\left(i_{n}\right)
$$

that is, we treat the distributions of the nodes as independent quantities. This approximation allows us to write the cloud for node $k$ as a product of independent bridge distributions originating from their respective origin node clouds:

$$
\begin{aligned}
& P_{k}(i) \approx \frac{\text { const }}{w(i)^{n-1}} \prod_{l=1}^{n} \sum_{i_{l}} P_{l}\left(i_{l}\right) K_{N_{b, l}}\left(i_{l}, i\right) \\
& =\frac{\text { const }}{w(i)^{n-1}} \prod_{l=1}^{n} W_{N_{b, l}}(i)
\end{aligned}
$$

which is eq 4. We derive this form in more detail in the Supporting Information (section SI).

To compute the ensemble-averaged monomer density in the system, we compute the normalized spatial probability distribution $\rho_{b, m}(i)$ of each bridge monomer $m$. Suppose that monomer $m$ is in bridge $b$, connecting nodes $j$ and $k$. (Note that monomer $m$ is the $m$ th segment in the bridge of $N_{b}$ segments.) The un-normalized form of the spatial probability distribution for monomer $m$ in the bridge is derived in the Supporting Information (section SII); it reads

$$
\begin{aligned}
& \rho_{b, m}^{\prime}(i)=\frac{1}{w(i)}\left[\sum_{i_{j}} P_{j}\left(i_{j}\right) \frac{K_{m}\left(i_{j}, i\right)}{\sum_{i_{k}} P_{k}\left(i_{k}\right) K_{N_{b, l}}\left(i_{k}, i_{j}\right)}\right] \\
& \times\left[\sum_{i_{k}} P_{k}\left(i_{k}\right) \frac{K_{N_{b, l}-m+1}\left(i_{k}, i\right)}{\sum_{i_{j}} P_{j}\left(i_{j}\right) K_{N_{b, l}}\left(i_{j}, i_{k}\right)}\right]
\end{aligned}
$$

where $K_{m}\left(i_{j}, i\right)$ is the sum of propagator weights for going from site $i_{j}$ to $i$ in $m$ steps, and $K_{N_{\mathrm{b},}-m+1}\left(i_{j}, i\right)$ is that for going from site $i_{k}$ to $i$ in $N_{b, l}-m+1$ steps. This distribution must normalize to unity, so that we have

$$
\rho_{b, m}(i)=\frac{\rho_{b, m}^{\prime}(i)}{\sum_{i^{\prime}} \rho_{b, m}^{\prime}\left(i^{\prime}\right)}
$$

The quantity $\rho_{b, m}(i)$ is the normalized spatial probability distribution for monomer $m$.

To obtain the total monomer density field, for all bridges $b$ we sum over the distributions for each segment in the bridge, except the two terminal segments as these are monomers potentially shared by multiple bridges. We then add in the $J$ node segments separately to prevent overcounting. Mathematically, this reads

$$
\rho(i)=\sum_{b} \sum_{m=2}^{N_{b}-1} \rho_{b, m}(i)+\sum_{j=1}^{J} P_{j}(i)
$$

The clouds $P_{j}(i)$ for each of the $J$ nodes in the system are not known a priori, nor is the ensemble-average number of monomers $\rho(i)$ in each site $i$. We therefore obtain these quantities by self-consistent iteration, starting with initial guesses.

Remarkably, for the networks examined here, we converged on $\rho(i)$ and each $P_{j}(i)$ starting from completely naive (uniform) distributions; that is, prior information about the spatial forms of $\rho(i)$ and the $P_{j}(i)$ is not required. 
We initialize $P_{j}^{\text {init }}(i)=1 / N_{\text {sites }}$ for all nodes $j$, and $\rho^{\text {init }}(i)=$ $\sum_{b} N_{b} / N_{\text {sites }}$, where $N_{\text {sites }}$ is the number of lattice sites in the system, and the sum in the second equation is over all bridges $b$. Given initial guesses for $\rho(i)$ and all $P_{j}(i)$, we then iterate to find self-consistent solutions. To improve stability, we use an adjustable blending parameter $\lambda$ that in each iteration, interpolates between "old" and "calculated" values to find the "new" values, through

$$
P_{j}^{\text {new }}(i)=\lambda P_{j}^{\text {calc }}(i)+(1-\lambda) P_{j}^{\text {old }}(i)
$$

for all nodes $j$

$$
\rho^{\text {new }}(i)=\lambda \rho^{\text {calc }}(i)+(1-\lambda) \rho^{\text {old }}(i)
$$

The "calculated" distributions $\rho(i)^{\text {calc }}$ and $P_{j}^{\text {calc }}(i)$ are those that directly result from eqs 4 and 10 using $\rho(i)^{\text {old }}$ and $P_{j}^{\text {old }}(i)$ (or, on the first iteration, $P_{j}^{\text {init }}(i)$ and $\left.\rho^{\text {init }}(i)\right)$ as input. The "new" distributions then become the "old" ones on the next iteration cycle.

The iteration is continued until the new distributions are equal to the input (old) distributions, within a small margin of error. Here, we iterate until the total variance between the "old" and "new" monomer density fields $\rho(i)$ first drops below $10^{-10}$. The blending coefficient $\lambda$ is tuned to control the numerical rate and accuracy of convergence; however, it does not affect the self-consistent solutions for $P_{j}(i)$ and $\rho(i)$.

To assess the computational scaling of our model, we perform a series of calculations on networks with different numbers of bridges and different lattice sizes. Compute time results are shown in Figures S2 and S3. Calculations are performed on one core of a standard central processing unit (CPU). As expected, the compute time per iteration scales linearly with the number of bridges as well as the number of sites in the lattice. We have also developed a parallelized version of our model in the Nvidia CUDA framework, which runs on Nvidia graphics cards (GPUs). Preliminary benchmarks in Figures S2 and S3 show that the GPU code is significantly faster than the CPU implementation on a modern mediumrange card (Nvidia GTX 1070). The speed-up grows larger for systems with more bridges and even more for systems in larger lattices. For example, note the small slope of the linear fit of the GPU results in Figure S2 (lower right) compared to that of the CPU results in the lower left panel.

To summarize, our model has the following input: the number of nodes, J; the network topology (i.e., the connectivity between the nodes); the number of segments $N_{b}$ comprising each connection (bridge); and the monomer interaction parameter, $\epsilon$. The latter can be zero, leading the polymer bridges to behave as ideal chains. The lattice model selfconsistently builds up the equilibrium ensemble of polymer chain conformations and node positions that satisfy the imposed network topology and monomer interaction parameter $\epsilon$. The result is the equilibrium spatial probability distributions, or "clouds", for each of the free nodes in the network, as well as the equilibrium monomer density field.

\section{RESULTS}

In this section, we demonstrate an application of our SCFT model to a polymer network at rest and when sheared. The model yields the spatial probability distribution ("cloud") for each network node, which are compared to molecular dynamics simulations for validation. The entropy of each node is extracted, revealing how each uniquely varies as the network is sheared.

To study the role of node entropy in the deformation free energy of a network, we present results on a sequence of three additional networks, differing in the average number of connections per node. By comparing the free energy of each network as it is deformed, we identify to what extent node positional entropy affects the deformation pathway and how the pathway deviates from the classical rubber elasticity theory prediction.

We start our discussion of results with an application to the network topology shown in Figure 1. Calculations are carried

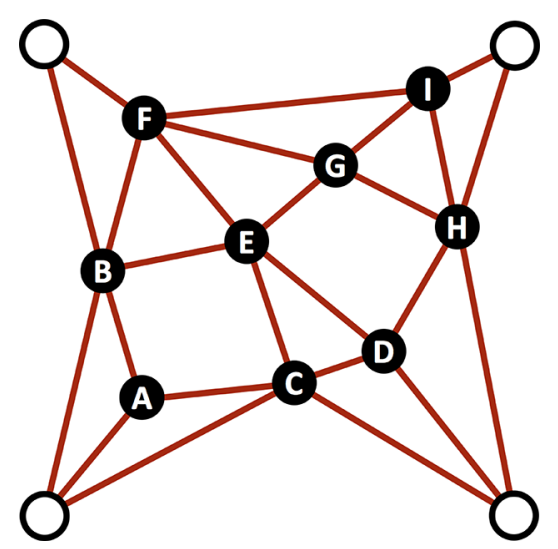

Figure 1. Connectivity diagram for a test network. Solid points are (free) nodes, open points are fixed nodes, and lines are bridges. Number of segments $N_{b}$ in each bridge is proportional to the squared length of the bridge in this diagram (see text for more details).

out on the network in its native state as well as a sequence of sheared configurations. In all cases, the monomer interaction parameter $\epsilon=0$, so that the bridges are noninteracting finitelength lattice polymers. When $\epsilon=0$, only the node clouds $P_{j}(i)$ need to be self-consistently calculated, while the monomer density field is free as the propagators do not interact with it.

As illustrated in Figure 1, the four corner nodes of the network are fixed, while the remaining nodes are free to fluctuate. The number of segments in each bridge is set to $N_{b}=$ floor $\left(s \times l_{b}^{2}\right)$, where $l_{b}$ is the length of the given bridge $b$ in Figure 1 given that the width and height of the illustration are both unity. A single monomer in the lattice is defined to have a diameter of unity, which is equal to the lattice constant. The parameter $s$ thus uniformly inflates or deflates the available contour length in each polymer bridge in the network. For example, at a given system size and fixed corner node positions, smaller $s$ leads to fewer monomers per chain, and thus a network that is under greater internal tension.

To perform shear, the two upper fixed nodes of the network are displaced by some amount $\Delta x_{\text {shear }}$ while keeping the two lower fixed nodes in their original positions. The resulting strain is $\gamma=\Delta x_{\text {shear }} / h$, where $h$ is the height of the network.

To assess the accuracy of the SCFT approach, results are compared to molecular dynamics (MD) simulations of a twodimensional polymer network with the same topology and bridge lengths. The bridges are modeled as noninteracting bead-rod polymers, with monomers of diameter $\sigma=1$, mass unity, and fixed bond lengths of unity (all in simulation units). Nodes are identical to monomers in the bridges, though they can have more than two bonds. Fixed nodes are defined to be monomers having fixed coordinates in the simulation box. The 
system is integrated using Langevin dynamics with $k_{\mathrm{B}} T=1$, a friction coefficient of unity for all particles, and a time step size of $\mathrm{d} t=0.001$ (in simulation time units). The simulation box is periodic in both dimensions; however, the box size is set to be large enough so that the system statistics do not include the images of the network across the boundaries. Systems are initially equilibrated for $10^{5}$ time steps, and then statistics are taken and averaged over $10^{8}$ time steps. The HOOMD-Blue package was used to perform the calculations. ${ }^{28,29}$

We now examine the performance of our SCFT model on the native (unsheared) network. The network is chosen to have a width and height of 50 lattice units (i.e., monomer diameters). Like in the MD simulation, the total lattice size is chosen to be much larger than this to prevent the system statistics from including images of the network across the reflecting boundary conditions. The number of segments in each bridge is set to $N_{b}$ $=$ floor $\left(s \times l_{b}^{2}\right)$. For the results reported here in the main text, the scaling parameter $s=50$. Comparison between the model and simulation for another choice, $s=100$, is given in the Supporting Information and described shortly.

Figure 2 presents results from the SCFT model for the clouds of selected nodes A, D, F, and I (see Figure 1). Overlaid

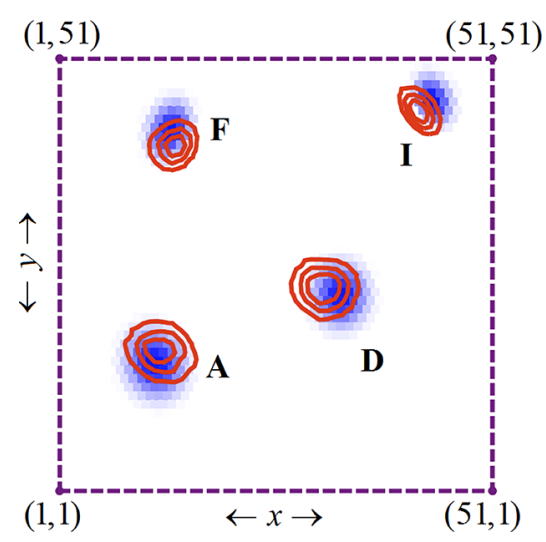

Figure 2. Spatial probability distributions for selected free nodes A, D, $\mathrm{F}$, and I indicated in Figure 1, in the unsheared network with $s=50$. Blue shading is prediction from our lattice model normalized to the largest value on the lattice; shading intensity is continuous from values 0 (white) to 1 (blue). Red contours are interpolated results obtained from molecular dynamics simulation; contours are drawn at values of $1 / 4,1 / 2$, and $3 / 4$. Four fixed nodes in the network are plotted as purple points, located at lattice coordinates indicated by ordered pairs. These are connected for visual convenience by purple dashed lines.

on the model output are results from the corresponding MD simulation. We find that the clouds obtained from the lattice model are in good quantitative agreement with those obtained from simulation. We again emphasize here: the lattice model results were obtained without any prior knowledge of the equilibrium distribution of the nodes in the system. Results for all nodes in the network, in unsheared $(\gamma=0)$ and sheared $(\gamma=$ 1) states, are given for $s=50$ and $s=100$ in Figure $S 4$.

To more closely assess the accuracy of our model upon shear, we plot the ensemble-average $x$ and $y$ coordinate of nodes A, D, F, and I as a function of strain $\gamma$ in Figure 3. Dashed lines in that figure indicate the predicted positions of the nodes if they were to deform affinely with the shear. Results for the remaining nodes in the network are given in Figure S5. Deviation of the node positions from the affine prediction, particularly in the $y$ direction (i.e., perpendicular to the shear axis), arises due to the finite lengths of the polymers in this simple case. This becomes an important contribution to the ensemble of network microstates at large strain, to be revisited shortly.

The results from the lattice model (solid curves) fall close to what is observed in simulation (points connected by dotted lines) even up to the substantial shear strain of $\gamma=1$. Importantly, the deviation of the lattice model results from simulation is in large part constant with strain. Thus, nonaffine displacement of nodes in the MD simulations is also captured to a reasonable extent by the lattice model, particularly the nontrivial shift in $y$ coordinates (i.e., perpendicular to the shear axis $x$ ) in Figure 3 (middle).

The entropy of a node depends on the spatial extent of its cloud $P_{j}(i)$, which in turn depends on how strongly it is enslaved to the network as a whole. For a given network topology, the SCFT model yields the full equilibrium ensemble of chain conformations and node positions. The entropy of a node $j$ can be easily extracted by the Gibbs expression:

$$
\frac{S_{j}}{k_{\mathrm{B}}}=-\sum_{i} P_{j}(i) \ln P_{j}(i)
$$

Figure 3, right panel, plots the entropy of nodes A, D, F, and I in the test network as a function of shear. Nodes have both positive and negative changes in entropy upon shear, and the magnitude of the change also varies across nodes depending on their location in the network. For example, node F initially gains entropy, as the distance between the upper left and lower right corner of the network grows smaller as it is sheared toward $\gamma=$ 1.

In Figures S4, S6, and S7, the scaling factor $s$ is doubled to 100. Doing so causes each bridge in the network to be composed of double the number of segments. In Figure S4, the longer chains allow each node to explore more space, resulting in larger node clouds and overall larger entropies per node in Figures S6 and S7. However, the longer strands cause the network to deform more affinely due to their larger finite lengths (i.e., they behave like ideal Gaussian chains up to larger end-to-end extensions). The network also exhibits a smaller variation in node entropy as the network is sheared. Like in the $s=50$ case, we find good agreement between our model, and the molecular dynamics simulations.

The nontrivial changes in node entropy and spatial distribution upon deformation are an important prediction of our model. This is because the model explicitly enforces the connectivity of the network according to the input topology. The key approximation in making the model computationally tractable is to solve for the node spatial distributions independently via eq 6; however, this approximation clearly retains the essential behavior of the nodes upon deformation.

We now examine to what extent node entropy affects the overall free energy change of a network as it is deformed, depending on the connectivity of the network. In the SCFT model, the free energy of the network can be approximated as the sum of the free energies of all the bridges (noting that this overcounts the contribution from the nodes, i.e., the bridge end points). The free energy of a bridge of $N_{b}$ segments connecting nodes $j$ and $k$ is

$$
F_{b} / k_{\mathrm{B}} T=-\ln \left(\sum_{i} P_{k}(i) W_{N_{b}}(i)\right)
$$



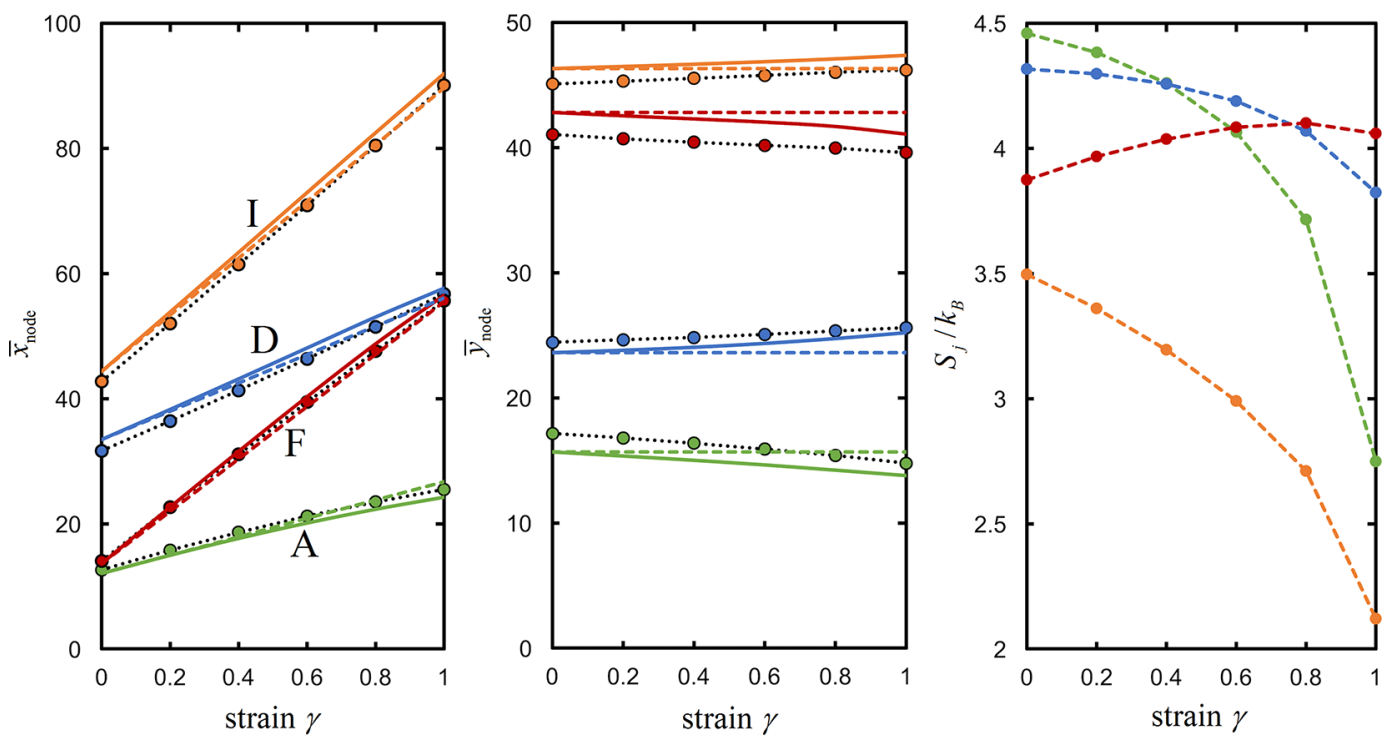

Figure 3. Mean $x$ (left panel) and $y$ (middle panel) coordinates of nodes A, D, F, and I as a function of strain $\gamma$ when $s=50$. Solid lines are results from our lattice model, dashed lines are displacements expected in the affine limit, and points connected by dotted lines are MD simulation results. Right panel shows node entropy from our lattice model, as a function of strain $\gamma$; points are lattice model results, and lines are guides for the eye.
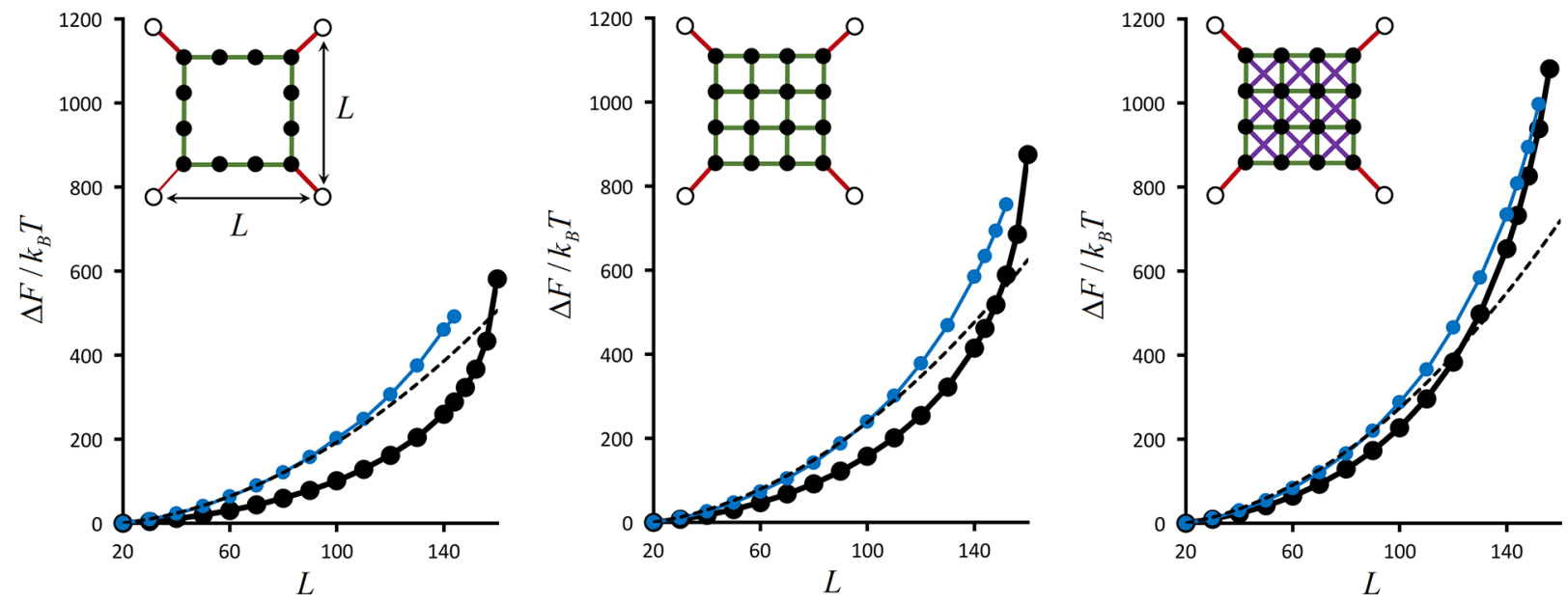

Figure 4. Network free energy (eq 15, black points) and free energy when nodes are fixed to their most probable positions (eq 18, blue points) as a function of network size $L$ (width = height, in lattice units). Results are given for three different networks, with connectivities given by the inset in each plot. Dashed black lines are predictions from the classical rubber elasticity theory. Curves are shifted so that all free energies are zero for $L=20$ lattice units. Black and blue points are connected by fit lines as guides for the eye. (insets) Connectivity diagram for each network, showing fixed nodes (open points), free nodes (solid points), and bridges (lines). Line color represents the number of monomers composing the given bridge: red is 50; green is 33 , and purple is 66 .

where the term in parentheses is the partition function for chain conformations that begin within the cloud of node $j$ and end in that of node $k$. Note that the termination points for the bridge in the cloud for node $k$ are properly weighted by $P_{k}(i)$. Moreover, via eqs 1 and 2, $W_{N_{b}}(i)$ contains the proper weights $P_{j}(i)$ for starting chain conformations at various points in the node $j$ cloud. The free energy of the network is then

$$
F=\sum_{\text {bridges } b} F_{b}
$$

To assess the contribution of node positional entropy to the network free energy, we compare to the hypothetical case in which nodes are fixed to their most probable positions $i_{j}^{*}$. The free energy $F_{b}^{*}$ for a bridge in this case is

$$
F_{b}^{*} / k_{\mathrm{B}} T=-\ln \left(P_{k}\left(i_{k}^{*}\right) W_{N_{b}}^{*}\left(i_{k}^{*} ; i_{j}^{*}\right)\right)
$$

Here, the modified propagator weight $W_{N b}^{*}\left(i_{k}^{*} ; i_{j}^{*}\right)$ is initialized in eq 1 with

$$
\begin{aligned}
& p_{1}(i)=P_{j}(i) \quad \text { if } i=i_{j}^{*} \\
& =0 \text { otherwise }
\end{aligned}
$$

The total network free energy when nodes are fixed to their most probable positions is therefore

$$
F^{*}=\sum_{\text {bridges } b} F_{b}^{*}
$$

Figure 4 examines the free energy change of three different twodimensional networks when they are isotropically expanded from their corners. Connectivity diagrams of the networks are shown as insets in Figure 4, illustrating fixed nodes, free nodes, and bridges. Isotropic expansion of the network is carried out 
by changing the distance $L$ between the fixed nodes on the lattice, such that the width and height of the network are always equal. The "true" (SCFT model) free energy of the network (eq 15) is compared to that when nodes are fixed to their most probable positions for a given $L$ (eq 18). Predictions from the classical rubber elasticity theory ${ }^{18}$ are also overlaid; details of this calculation are given in the Supporting Information.

The black points connected by bold lines in Figure 4 are the true free energy change of the networks upon expansion. Increasing the number of bridges in the system, moving from left to right in the figure panels, has the obvious effect of increasing the free energy required to expand the network. This is the collective "entropic elasticity" of the polymer chains. Adding more polymers to the network increases the (entropic) work required to increase the end-to-end distances of the chains. At large strains, chains approach being stretched to their maximum end-to-end length. The free energy therefore increases more drastically.

The translational entropy of the nodes (cross-links) themselves play a significant role in the network free energy as it is expanded. Indeed, if we "freeze out" the nodes such that they are localized to their most probable positions, the free energy cost to expand the network is larger. As shown by the blue data sets in Figure 4, this effect is particularly notable when the nodes have fewer bridges attached to them, as in the leftmost network in the figure. As the network is interconnected with more bridges (i.e., the right-most network in Figure 4), then each node intrinsically has less translational freedom. Isolating the nodes to their most probable positions therefore has less of an effect in changing the free energy landscape of the network as it is expanded.

As the networks are deformed to larger extents, nodes in the networks lose their translational entropy and are increasingly restricted to their most probable positions. Thus, the blue and black points in Figure 4 converge, indicating the loss of node translational entropy. This is also observed in Figure 3, where the entropy of all nodes approaches zero as the shear strain $\gamma$ increases.

Figure 4 also shows predictions for the free energy of deformation given by classical rubber elasticity theory. ${ }^{18}$ In the theory, the only free energy contribution to deformation arises from stretching the polymers. The polymers are assumed to be purely Gaussian, and the nodes are assumed to be localized to their most probable positions while deforming affinely with the overall deformation of the network. Thus, for small deformations, the classical elasticity theory naturally correlates with the blue curves in Figure 4, i.e., the SCFT calculations in which nodes are localized to their most probable positions. The correlation also confirms that the network is deforming affinely in this regime within the lattice model. At larger deformations, chains approach being stretched to their maximum end-to-end lengths in the lattice model, leading to a sharper growth in the network free energy compared to that predicted by the purely Gaussian elasticity theory.

\section{CONCLUSIONS}

Polymers connected into a network exhibit complex conformational behavior. How the conformational space for each polymer in the network evolves upon deformation depends in a nontrivial way on the network topology. Other ingredients, such as interactions between the polymers, complicate this picture further.
With this in mind, we have developed a simple lattice model based on polymer self-consistent field theory, with the goal of having a tool that efficiently samples the equilibrium statistics of an arbitrary polymer network. A potent aspect of our model is that it is able to generate the complete equilibrium ensemble of finitely extensible polymer conformations and cross-link positions on a lattice, without any prior information as to what these should be. Cross-link entropy can be easily extracted from the model results and nonaffinity of deformation assessed.

To make the model computationally feasible, we decouple the node spatial distributions into independent fields that nevertheless satisfy the input polymer network topology. This approximation leads to a simple model that captures the salient variations in node entropy and spatial fluctuations upon deformation. Indeed, the results of our model are in close agreement with molecular dynamics simulation, including when the polymer networks are sheared to high strains. Albeit at the expense of some molecular complexity, our model is well suited to studying large polymer networks without the kinetic barriers and computational limitations often encountered in a full molecular simulation.

As an example, we have shown how our approach can be used to compute the deformation "free energy landscape" of a polymer network that intrinsically captures the unique translational entropy of each cross-link. This leads to deformation free energy landscapes that are quite different from those expected by classical rubber elasticity theory. Indeed, our model presents an advantage over more simplified mean-field approaches to polymer networks, given that it has topological specificity and provides spatial resolution of the polymer network statistics.

At present, the model does not account for chain noncrossing and entanglements. We anticipate adding this ingredient into the model by a cross-link species that can bind, slide, and unbind along the participating polymer chains. Meanfield monomer incompressibility, using a conjugate chemical potential field, ${ }^{30}$ can also be naturally incorporated into our approach; however, it is likely this would necessitate a more sophisticated method for finding the self-consistent solution to the model equations.

The simplicity of our approach makes it amenable to including more complex molecular ingredients. Because it is based on self-consistent field theory, our model can borrow from the vast body of research that has already been done on that method. The computational efficiency of the model particularly on GPUs also makes it well-suited to studying very large networks while retaining microscopic detail. We believe this will be useful for studying chain failure and structural change in strongly deformed polymer networks along with recent experimental realizations of dynamic network bonding, e.g., in vitrimers, ${ }^{7}$ self-healing polymers, ${ }^{2}$ and reversibly crosslinked materials. ${ }^{5}$

\section{ASSOCIATED CONTENT}

Supporting Information

The Supporting Information is available free of charge on the ACS Publications website at DOI: 10.1021/acs.macromol.7b01284.

Computational scaling of model, additional results, and detailed derivation of key equations in the main text (PDF) 


\section{AUTHOR INFORMATION}

\section{Corresponding Author}

*E-mail: nicholas.b.tito@gmail.com (N.B.T.).

\section{ORCID $\odot$}

Nicholas B. Tito: 0000-0002-8602-012X

Wouter G. Ellenbroek: 0000-0002-9336-6233

\section{Notes}

The authors declare no competing financial interest.

\section{ACKNOWLEDGMENTS}

This research has been performed within the framework of the 4TU.High-Tech Materials research programme "New Horizons in designer materials". We are grateful for compute time obtained from The Netherlands Organisation for Scientific Research (NWO) SURFsara Pilot Program (Grant No. 15582). Additional thanks to Anwesha Bose, Stefano Angioletti-Uberti, Chiara Raffaelli, and Simone Ciarella for valuable discussions.

\section{REFERENCES}

(1) Lee, K. Y.; Mooney, D. J. Hydrogels for Tissue Engineering. Chem. Rev. 2001, 101, 1869-1880.

(2) Zwaag, S. v. d.; Brinkman, E. Self Healing Materials; IOS Press: Amsterdam, The Netherlands, 2015.

(3) Stukalin, E. B.; Cai, L.-H.; Kumar, N. A.; Leibler, L.; Rubinstein, M. Self-Healing of Unentangled Polymer Networks with Reversible Bonds. Macromolecules 2013, 46, 7525-7541.

(4) White, T. J.; Broer, D. J. Programmable and adaptive mechanics with liquid crystal polymer networks and elastomers. Nat. Mater. 2015, 14, 1087-1098.

(5) Kean, Z. S.; Hawk, J. L.; Lin, S.; Zhao, X.; Sijbesma, R. P.; Craig, S. L. Increasing the Maximum Achievable Strain of a Covalent Polymer Gel Through the Addition of Mechanically Invisible Cross-Links. Adv. Mater. 2014, 26, 6013-6018.

(6) Storm, C.; Pastore, J. J.; MacKintosh, F. C.; Lubensky, T. C.; Janmey, P. A. Nonlinear elasticity in biological gels. Nature 2005, 435, 191-194.

(7) Montarnal, D.; Capelot, M.; Tournilhac, F.; Leibler, L. Silica-Like Malleable Materials from Permanent Organic Networks. Science 2011, 334, 965-968.

(8) Broedersz, C. P.; MacKintosh, F. C. Modeling semiflexible polymer networks. Rev. Mod. Phys. 2014, 86, 995-1036.

(9) Escobedo, F. A.; DePablo, J. J. Phase behaviour of model polymeric networks and gels. Mol. Phys. 1997, 90, 437-443.

(10) Escobedo, F. A.; de Pablo, J. J. Molecular simulation of polymeric networks and gels: phase behavior and swelling. Phys. Rep. 1999, 318, 85-112.

(11) Huisman, E. M.; Storm, C.; Barkema, G. T. Monte Carlo study of multiply crosslinked semiflexible polymer networks. Phys. Rev. E 2008, 78, 051801.

(12) Buxton, G. A.; Clarke, N. Bending to Stretching" Transition in Disordered Networks. Phys. Rev. Lett. 2007, 98, 238103.

(13) Gordievskaya, Y. D.; Rumyantsev, A. M.; Kramarenko, E. Y. Polymer gels with associating side chains and their interaction with surfactants. J. Chem. Phys. 2016, 144, 184902.

(14) Erman, B.; Bahar, I.; Kloczkowski, A.; Mark, J. E. Lattice Model for Segmental Orientation in Deformed Polymeric Networks 0.1. Contribution of Intermolecular Correlations. Macromolecules 1990, 23, $5335-5340$.

(15) Holzl, T.; Trautenberg, H. L.; Goritz, D. Monte Carlo simulations on polymer network deformation. Phys. Rev. Lett. 1997, 79, 2293-2296.

(16) Gurtovenko, A. A.; Blumen, A. Relaxation of disordered polymer networks: Regular lattice made up of small-world Rouse networks. J. Chem. Phys. 2001, 115, 4924-4929.
(17) Gilra, N.; Panagiotopoulos, A. Z.; Cohen, C. Monte Carlo Simulations of Polymer Network Deformation. Macromolecules 2001, $34,6090-6096$

(18) Rubinstein, M.; Colby, R. H. Polymer Physics; Oxford University Press: 2003

(19) Xing, X.; Goldbart, P. M.; Radzihovsky, L. Thermal Fluctuations and Rubber Elasticity. Phys. Rev. Lett. 2007, 98, 075502.

(20) Rubinstein, M.; Leibler, L.; Bastide, J. Giant Fluctuations of Cross-Link Positions in Gels. Phys. Rev. Lett. 1992, 68, 405-407.

(21) Barriere, B. Elastic-Moduli of 2d Grafted Tethered Membranes. J. Phys. I 1995, 5, 389-398.

(22) Scheutjens, J. M. H. M.; Fleer, G. J. Statistical theory of the adsorption of interacting chain molecules. 1. Partition function, segment density distribution, and adsorption isotherms. J. Phys. Chem. 1979, 83, 1619-1635.

(23) Schmid, F. Self-Consistent Field Approach for Cross-Linked Copolymer Materials. Phys. Rev. Lett. 2013, 111, 028303.

(24) Leermakers, F. A. M.; Scheutjens, J. M. H. M. Statistical Thermodynamics of Association Colloids 0.1. Lipid Bilayer-Membranes. J. Chem. Phys. 1988, 89, 3264-3274.

(25) Bergsma, J.; Leermakers, F. A. M.; van der Gucht, J. Interactions between nodes in a physical gel network of telechelic polymers; selfconsistent field calculations beyond the cell model. Phys. Chem. Chem. Phys. 2015, 17, 9001-9014.

(26) DiMarzio, E. A.; Rubin, R. J. Adsorption of a Chain Polymer between Two Plates. J. Chem. Phys. 1971, 55, 4318-4336.

(27) de Gennes, P.-G. Scaling Concepts in Polymer Physics; Cornell University Press: 1979

(28) Anderson, J. A.; Lorenz, C. D.; Travesset, A. General purpose molecular dynamics simulations fully implemented on graphics processing units. J. Comput. Phys. 2008, 227, 5342-5359.

(29) Glaser, J.; Nguyen, T. D.; Anderson, J. A.; Lui, P.; Spiga, F.; Millan, J. A.; Morse, D. C.; Glotzer, S. C. Strong scaling of generalpurpose molecular dynamics simulations on GPUs. Comput. Phys. Commun. 2015, 192, 97-107.

(30) Fredrickson, G. H. Equilibrium Theory of Inhomogenous Polymers: Oxford University Press: 2006. 